\title{
Determinants of Attrition to Follow-Up in a Multicentre Cohort Study in Children-Results from the IDEFICS Study
}

\author{
Sabrina Hense, ${ }^{1}$ Hermann Pohlabeln, ${ }^{2}$ Nathalie Michels, ${ }^{3}$ Staffan Mårild, ${ }^{4}$ \\ Lauren Lissner, ${ }^{5}$ Eva Kovacs, ${ }^{6}$ Luis A. Moreno, ${ }^{7}$ Charalampos Hadjigeorgiou, ${ }^{8}$ \\ Toomas Veidebaum, ${ }^{9}$ Licia Iacovello, ${ }^{10}$ Yannis Pitsiladis, ${ }^{11}$ Lucia Reisch, ${ }^{12}$ \\ Alfonso Siani, ${ }^{13}$ and Wolfgang Ahrens ${ }^{14}$ \\ ${ }^{1}$ Department of Clinical Epidemiology, Leibniz Institute for Prevention Research and Epidemiology, BIPS, Germany \\ ${ }^{2}$ Department of Biometry and Data Management, Leibniz Institute for Prevention Research and Epidemiology, BIPS, Germany \\ ${ }^{3}$ Department of Public Health, Faculty of Medicine and Health Sciences, Ghent University, Belgium \\ ${ }^{4}$ Department of Paediatrics, Institute of Clinical Sciences, Sahlgrenska Academy at University of Gothenburg, Sweden \\ ${ }^{5}$ Department of Public Health and Community Medicine, University of Gothenburg, Sweden \\ ${ }^{6}$ Department of Pediatrics, Medical Faculty, University of Pécs, Hungary \\ ${ }^{7}$ GENUD Research Group, Facultad de Ciencias de la Salud, University of Zaragoza, Spain \\ ${ }^{8}$ Research and Education Institute of Child Health, Cyprus \\ ${ }^{9}$ National Institute for Health Development, Estonia \\ ${ }^{10}$ Fondazione di Ricerca e Cura Giovanni Paolo II, Università Cattolica, Campobasso, Italy \\ ${ }^{11}$ College of Medicine, Veterinary \& Life Sciences, Institute of Cardiovascular \& Medical Sciences, University of Glasgow, UK \\ ${ }^{12}$ Department of Intercultural Communication and Management, Copenhagen Business School, Denmark \\ ${ }^{13}$ Unit of Epidemiology \& Population Genetics, Institute of Food Sciences, National Research Council, Avellino, Italy \\ ${ }^{14}$ Department of Epidemiological Methods and Etiologic Research, Leibniz Institute for Prevention Research and Epidemiology, \\ BIPS, University of Bremen, 28359, Germany
}

Correspondence should be addressed to Wolfgang Ahrens; ahrens@bips.uni-bremen.de

Received 10 January 2013; Accepted 13 April 2013

Academic Editor: Demosthenes Panagiotakos

Copyright (C) 2013 Sabrina Hense et al. This is an open access article distributed under the Creative Commons Attribution License, which permits unrestricted use, distribution, and reproduction in any medium, provided the original work is properly cited.

Cohort participant retention is a crucial element and may depend on several factors. Based on data from a multicentre cohort of
European children, the effect of baseline participation on attrition and the association with and the impact of single determinants
in relation to the extent of attrition were investigated. Data was available for 16,225 children from the IDEFICS baseline survey
(2007/2008). Attrition was defined as nonparticipation in the first follow-up examination $(2009 / 2010)$. Determinants of attrition
were analysed by logistic regression. The statistical significance level was set at $\alpha=0.01$ to account for the large sample size.
The strongest associations were seen for baseline item non-response, especially when information on migration background
(odds ratio (OR) =1.55; $99 \%$ confidence interval (CI): $1.04,2.31)$, single parenthood (OR = 1.37; $99 \%$ CI: 1.12, 1.67), or well-being
$(\mathrm{OR}=1.46 ; 99 \% \mathrm{CI}: 1.19,1.79)$ was lacking. Drop-out proportion rose with the number of missing items. Overweight, low
education, single parenthood and low well-being scores were independent determinants of attrition. Baseline participation, and
the individual determinant effects seemed unrelated to the variation of the extent of attrition between study centres. A high level
of item nonresponse as well as overweight and disadvantageous sociodemographic conditions were identified as main attrition
determinants, suggesting the consideration of these aspects in conduct and analysis of cohort studies in childhood obesity research.

\section{Introduction}

In longitudinal studies, participant adherence is a crucial element of study organisation that requires considerable effort and time. However, attrition is an inevitable problem in almost every epidemiological cohort study, which leads to a loss of power and potentially introduces selection bias when drop-out is related to the exposures or outcomes of 
interest. The most commonly reported types of attrition are noncontact or refusal [1]. In fact, the motivation of study participants to remain involved in the study activities may decline over time and will depend on several factors. To apply appropriate analysis strategies and to allow for a correct interpretation of the results from longitudinal studies, knowledge on the determinants of attrition is important. Additionally, information about factors affecting attrition is crucial to improve retention of cohort participants. One aspect that has been reported to affect attrition is baseline response and concomitant recruitment efforts [2]. With regard to longitudinal studies in children and adolescents, where parental consent is commonly required, low parental education level, migration background, and single parenthood have been suggested to mainly determine attrition rates $[3,4]$. However, there is little experience regarding the effects of different determinants of attrition in a multicentre study where the same standardized study protocols are applied. Such data would facilitate the comparison between study centres that have different attrition proportions and baseline responses and the extent of potential selection effects.

This report presents results regarding the proportionate attrition from baseline (T0) to the first follow-up examination (T1) of the multi-centre IDEFICS (identification and prevention of dietary- and lifestyle-induced health effects in children and infants) study. We investigated systematic drop-out related to weight status as well as to known obesity risk factors, such as low educational level and migration background. Also, behavioural factors that have been identified as determinants of overweight, such as screen time [5], sleep duration [6], or dietary behaviour [7, 8], and certain psychosocial aspects [9] were examined as possible attrition determinants. Selective drop-out with regard to these factors might affect the interpretability of results in longitudinal studies of obesity research. Additionally, allocation to either the intervention or the control region (see the following for more details) might play a role in this context and was therefore another determinant of interest in the present study.

In this paper we (1) assess determinants of attrition and evaluate whether (2) the baseline participation in the study centres is associated with attrition and (3) whether the impact of attrition determinants is stronger in centres with a high proportionate attrition.

\section{Methods}

2.1. Study Population. The IDEFICS project is a populationbased multicentre intervention study which included children aged 2 to 9 years from eight European countries.

Between September 2007 and May 2008, 31543 children from schools and preschools in selected regions in Italy, Estonia, Cyprus, Belgium, Sweden, Hungary, Germany, and Spain were asked to participate in the baseline survey (T0) based on a two-stage random sampling strategy with schools presenting the first and classes presenting the second level of sampling units. Of those children 16864 (53.4\%) accepted the invitation, with 16225 (51.4\%) providing the parental questionnaire and measurement of weight and height [10]. They thus fulfilled the preset inclusion criteria and were eligible for the present analysis. The baseline survey was followed by a community- and setting-based intervention program concerning diet, physical activity, and stress coping, which was implemented in intervention regions in each of the participating countries, while no intervention was offered in the control regions [11]. Two years later, every child who had participated in the T0 survey was automatically reinvited to take part in the T1 survey (September 2009 to June 2010). Both, T0 and T1 involved a similarly extensive study protocol including anthropometric examinations, collection of biosamples (urine, saliva, blood), fitness, and sensory tests as well as questionnaires on diet, psychosocial, behavioural, and environmental factors to be filled in by the parents. More detailed information on the study protocol and procedures can be found elsewhere [12].

Two age groups were defined with one group including children aged 2 to $<6$ years (preschool children) and the other group including children aged 6 to 9 years (school children) at baseline.

In each country, the participating centres obtained ethical approval by their responsible authority. All children and their parents provided oral and written informed consent, respectively, for all examinations and/or the collection of samples, subsequent analysis, and storage of personal data and collected samples. More detailed information on the study design, applied instruments, and measurements as well as baseline response rates with regard to the respective study modules has been presented elsewhere [10].

\subsection{Measurements}

2.2.1. Attrition. The outcome measure was a dichotomous variable for attrition at two-year follow-up (T1 participant versus $\mathrm{T} 1$ nonparticipant). The amount of attrition per study centre was expressed as a percentage of the number of baseline participants.

2.2.2. Baseline Characteristics. All measurements were conducted according to a manual of standardized procedures in all centres. Body height was measured without shoes by trained staff using a portable stadiometer (SECA 225). Weight was measured by means of an adapted version of electronic scale TANITA BC 420 SMA, wearing only underwear. BMI was calculated and then categorized according to the criteria of the International Obesity Task Force [13]. Our reference category included normal and underweight children. Data on personal, social, environmental, and behavioural factors of each child, such as parental education level, family status, migration background, time spent in front of a TV or PC screen (screen time), and well-being was collected by means of a standardized parental self-completion questionnaire. Education level was categorized according to the International Standard Classification of Education (ISCED). Four levels of education (low versus medium-low versus mediumhigh versus high) were created out of the six ISCED levels, with ISCED levels $0-2$ being defined as low education, level 3 being defined medium-low, level 4 being defined as medium-high, and level 5 and higher being defined as high education. Migration background was defined as either mother 
or father of the child or both parents being born in a foreign country. Family status was defined as single versus two-parent families.

A well-being scale was developed, including information on emotional well-being, self-esteem, family, and friends. Though the scale itself was not validated, these questions were based on the respective subscales from the Kiddy$\mathrm{KINDL}^{\mathrm{R}}$ for parents of children aged $4-7$ years, a validated questionnaire for measuring health-related quality of life in children [14]. Answers were given according to a 4-point Likert scale (never, rarely, sometimes, often/all the time), that was adapted to the original 5-point Likert scale of the $\mathrm{KINDL}^{\mathrm{R}}$. Since the IDEFICS parental questionnaire did not comprise the whole subscale set from $\mathrm{KINDL}^{\mathrm{R}}$ (questions on physical well-being and everyday functioning were excluded in IDEFICS) no total instrument score, but only sum scores based on the included subscales were created as proposed by the authors of the original instrument [15]. Based on these scores we created four well-being categories (low, mediumlow, medium-high, and high). More detailed information on the $\mathrm{KINDL}^{\mathrm{R}}$ scores can be found at http://www.kindl.org/ [15].

Information on dietary habits was obtained from a validated standardized Food Frequency Questionnaire (CEHQFFQ) $[16,17]$. A variable representing the weekly consumption of junk food (including sweetened drinks, chocolate or nut based spreads, snacks) was created based on this data. Furthermore, the parents were asked if soft drinks were available during meals (yes versus no).

Sleep duration was assessed using a 24-hour dietary recall (SACINA). SACINA is a computer-based instrument that was filled out by parents or guardian of each participating child and contained questions on the time at which the child got up in the morning and went to bed on the previous day. Nocturnal sleep duration was calculated as the difference between bed and get-up time in the SACINA interview resulting in a continuous variable. More detailed information on the sleep duration assessment in IDEFICS is given elsewhere $[6,18]$. Two sleep duration categories were created ( $\leq 11$ hours of sleep versus $>11$ hours of sleep).

A "non-response-score" was developed, taking into account the amount of item non-response per participant with regard to the variables of the baseline survey, resulting in four categories: none (no item non-response); low (1 item non-response); medium (2 or more item non-responses).

Baseline participation rate was included in the analysis as a dichotomous variable per study centre with the categories low $(<50 \%)$ versus high $(\geq 50 \%)$.

2.3. Statistical Analysis. Descriptive analyses describe attrition proportion and sample characteristics in the participating study centres (countries). Chi square statistics were used for comparison of categorical variables. To analyse the association between potential determinants and individual attrition (as a dichotomous dependant variable) we used separate logistic regression models. More precisely, we applied the procedure GLIMMIX (SAS Institute, Cary, North Carolina, USA) and included adjustments for the second-level random

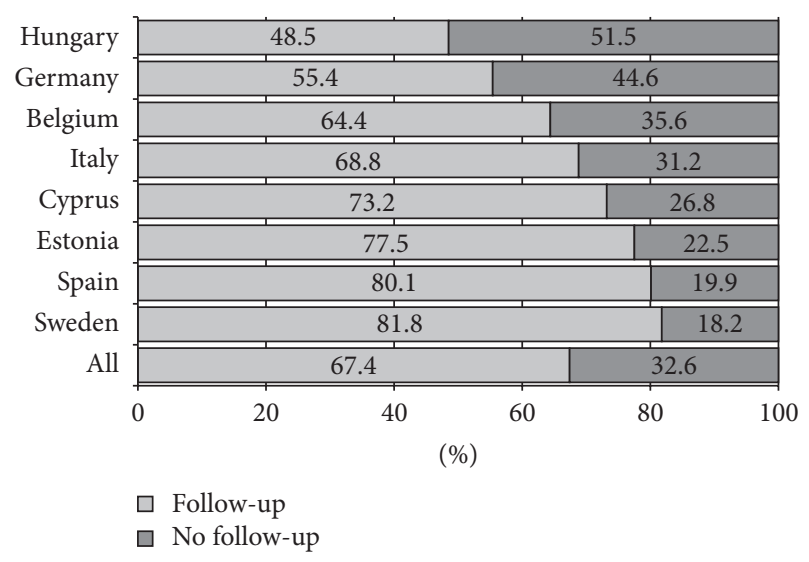

FIGURE 1: Attrition and participation proportion by country.

effect (country) as well as the simultaneous consideration of age and sex (Model 1). In a further step, we defined a multivariate logistic regression model (Model 2), which combined all variables showing a statistically significant effect, or an estimated odds ratio $(\mathrm{OR}) \geq 1.50$, in the separate Model 1 , to identify the strength and independence of the impact of single determinants on attrition. For sensitivity analysis, forward selection of covariates was additionally applied for model development to investigate if this approach would lead to inclusion of the same variables in the model. In order to compare whether the impact of determinants is related to the variation in the extent of attrition between study centres we conducted a country-stratified analysis (separate models for each country based on model 1), where country was used as a proxy for the degree of attrition. The statistical significance level was set at $\alpha=0.01$ to account for the large sample size.

\section{Results}

Among the 16225 children who participated in the IDEFICS baseline survey, the proportion of boys and girls was nearly equal in all survey centres, while slightly more school than preschool children participated in the survey in most countries, except for Sweden and Belgium. Striking country differences were seen in the percentage of obese and overweight children (e.g., Italy: $19.5 \%$ and $22.5 \%$; Belgium: $2.3 \%$ and $6.4 \%$, resp.) as well as in other potential determinants of attrition. Thus, for example, the proportion of children with migration background and parents with low educational level was very low in Estonia ( $4.6 \%$ and $1.9 \%$, resp.) as compared to Germany (34.1\% and $35.5 \%$, resp.). The proportion of single parents was low in Sweden (8.5\%) and relatively high in Italy (28.9\%). A detailed overview of all potential attrition determinants under investigation is given in Table 1.

The average proportionate attrition in the IDEFICS follow-up was $32.6 \%$, but it varied significantly between countries $(P<0.001)$, ranging from $18.2 \%$ in Sweden to $51.5 \%$ in Hungary (Figure 1).

Table 2 presents proportionate attrition and effect estimates for each category of the investigated factors applying two levels of adjustment (Models 1 and 2). Each potential 
TABLE 1: Baseline Response and Baseline Characteristics of Children in the IDEFICS Study by Country.

\begin{tabular}{|c|c|c|c|c|c|c|c|c|c|c|c|c|c|c|c|c|c|c|}
\hline & \multicolumn{2}{|c|}{ Estonia } & \multicolumn{2}{|c|}{ Sweden } & \multicolumn{2}{|c|}{ Germany } & \multicolumn{2}{|c|}{ Belgium } & \multicolumn{2}{|c|}{ Hungary } & \multicolumn{2}{|c|}{ Italy } & \multicolumn{2}{|c|}{ Spain } & \multicolumn{2}{|c|}{ Cyprus } & \multicolumn{2}{|c|}{ All } \\
\hline & $n$ & $\%$ & $n$ & $\%$ & $n$ & $\%$ & $n$ & $\%$ & $n$ & $\%$ & $n$ & $\%$ & $n$ & $\%$ & $n$ & $\%$ & $n$ & $\%$ \\
\hline \multicolumn{19}{|l|}{ Region } \\
\hline Intervention & 793 & $46.1 \%$ & 902 & $49.9 \%$ & 1179 & $57.1 \%$ & 976 & $50.7 \%$ & 1277 & $49.7 \%$ & 1182 & $52.5 \%$ & 798 & $53.0 \%$ & 1373 & $57.7 \%$ & 8480 & $52.3 \%$ \\
\hline Control & 926 & $53.9 \%$ & 907 & $50.1 \%$ & 886 & $42.9 \%$ & 950 & $49.3 \%$ & 1292 & $50.3 \%$ & 1068 & $47.5 \%$ & 709 & $47.0 \%$ & 1007 & $42.3 \%$ & 7745 & $47.7 \%$ \\
\hline \multicolumn{19}{|l|}{ Sex } \\
\hline Male & 850 & $49.4 \%$ & 934 & $51.6 \%$ & 1051 & $50.9 \%$ & 978 & $50.8 \%$ & 1286 & $50.1 \%$ & 1165 & $51.8 \%$ & 773 & $51.3 \%$ & 1226 & $51.5 \%$ & 8236 & $50.8 \%$ \\
\hline Female & 869 & $50.6 \%$ & 875 & $48.4 \%$ & 1014 & $49.1 \%$ & 948 & $49.2 \%$ & 1283 & $49.9 \%$ & 1085 & $48.2 \%$ & 734 & $48.7 \%$ & 1154 & $48.5 \%$ & 7962 & $49.1 \%$ \\
\hline \multicolumn{19}{|l|}{ Age } \\
\hline Pre-school & 859 & $50.0 \%$ & 918 & $50.7 \%$ & 876 & $42.4 \%$ & 1043 & $54.2 \%$ & 1039 & $40.4 \%$ & 974 & $43.3 \%$ & 715 & $47.4 \%$ & 954 & $40.1 \%$ & 7378 & $45.5 \%$ \\
\hline School & 860 & $50.0 \%$ & 891 & $49.3 \%$ & 1189 & $57.6 \%$ & 883 & $45.8 \%$ & 1530 & $59.6 \%$ & 1276 & $56.7 \%$ & 792 & $52.6 \%$ & 1426 & $59.9 \%$ & 8847 & $54.5 \%$ \\
\hline \multicolumn{19}{|l|}{ Weight status } \\
\hline Obese & 69 & $4.0 \%$ & 36 & $2.0 \%$ & 92 & $4.5 \%$ & 44 & $2.3 \%$ & 149 & $5.8 \%$ & 438 & $19.5 \%$ & 88 & $5.8 \%$ & 211 & $8.9 \%$ & 1127 & $6.9 \%$ \\
\hline Overweight & 178 & $10.4 \%$ & 160 & $8.8 \%$ & 237 & $11.5 \%$ & 123 & $6.4 \%$ & 288 & $11.2 \%$ & 507 & $22.5 \%$ & 223 & $14.8 \%$ & 345 & $14.5 \%$ & 2061 & $12.7 \%$ \\
\hline Normal weight & 1472 & $85.6 \%$ & 1613 & $89.2 \%$ & 1736 & $84.1 \%$ & 1759 & $91.3 \%$ & 2132 & $83.0 \%$ & 1305 & $58.0 \%$ & 1196 & $79.4 \%$ & 1824 & $76.6 \%$ & 13037 & $80.4 \%$ \\
\hline \multicolumn{19}{|c|}{ Migration background } \\
\hline No & 1572 & $91.4 \%$ & 1433 & $79.2 \%$ & 1304 & $63.1 \%$ & 1726 & $89.6 \%$ & 2398 & $93.3 \%$ & 1846 & $82.0 \%$ & 1310 & $86.9 \%$ & 1404 & $59.0 \%$ & 12993 & $80.1 \%$ \\
\hline Partly & 69 & $4.0 \%$ & 180 & $10.0 \%$ & 210 & $10.2 \%$ & 72 & $3.7 \%$ & 83 & $3.2 \%$ & 329 & $14.6 \%$ & 75 & $5.0 \%$ & 377 & $15.8 \%$ & 1395 & $8.6 \%$ \\
\hline Full & 11 & $0.6 \%$ & 146 & $8.1 \%$ & 493 & $23.9 \%$ & 42 & $2.2 \%$ & 19 & $0.7 \%$ & 54 & $2.4 \%$ & 86 & $5.7 \%$ & 240 & $10.1 \%$ & 1091 & $6.7 \%$ \\
\hline Missing & 67 & $3.9 \%$ & 50 & $2.8 \%$ & 58 & $2.8 \%$ & 86 & $4.5 \%$ & 69 & $2.7 \%$ & 21 & $0.9 \%$ & 36 & $2.4 \%$ & 359 & $15.1 \%$ & 746 & $4.6 \%$ \\
\hline \multicolumn{19}{|l|}{ Educational level } \\
\hline Low & 33 & $1.9 \%$ & 32 & $1.8 \%$ & 734 & $35.5 \%$ & 78 & $4.0 \%$ & 78 & $3.0 \%$ & 484 & $21.5 \%$ & 142 & $9.4 \%$ & 78 & $3.3 \%$ & 1659 & $10.2 \%$ \\
\hline Medium-low & 674 & $39.2 \%$ & 331 & $18.3 \%$ & 513 & $24.8 \%$ & 513 & $26.6 \%$ & 1107 & $43.1 \%$ & 1338 & $59.5 \%$ & 409 & $27.1 \%$ & 285 & $12.0 \%$ & 5170 & $31.9 \%$ \\
\hline Medium-high & 725 & $42.2 \%$ & 180 & $10.0 \%$ & 410 & $19.9 \%$ & 430 & $22.3 \%$ & 255 & $9.9 \%$ & 0 & $0.0 \%$ & 160 & $10.6 \%$ & 518 & $21.8 \%$ & 2678 & $16.5 \%$ \\
\hline High & 230 & $13.4 \%$ & 1215 & $67.2 \%$ & 321 & $15.5 \%$ & 850 & $44.1 \%$ & 1069 & $41.6 \%$ & 410 & $18.2 \%$ & 767 & $50.9 \%$ & 1144 & $48.1 \%$ & 6006 & $37.0 \%$ \\
\hline Missing & 57 & $3.3 \%$ & 51 & $2.8 \%$ & 87 & $4.2 \%$ & 55 & $2.9 \%$ & 60 & $2.3 \%$ & 18 & $0.8 \%$ & 29 & $1.9 \%$ & 355 & $14.9 \%$ & 712 & $4.4 \%$ \\
\hline 1 (4⿴囗十) & & & & & & & & & & & & & & & & & & \\
\hline Two par & 1414 & $82.3 \%$ & 1606 & $88.8 \%$ & 1675 & $81.1 \%$ & 1677 & $87.1 \%$ & 2036 & $79.3 \%$ & 1477 & $65.6 \%$ & 1322 & $87.7 \%$ & 1086 & $45.6 \%$ & 12293 & $75.8 \%$ \\
\hline Single parent & 214 & $12.4 \%$ & 153 & $8.5 \%$ & 309 & $15.0 \%$ & 184 & $9.6 \%$ & 394 & $15.3 \%$ & 651 & $28.9 \%$ & 132 & $8.8 \%$ & 391 & $16.4 \%$ & 2482 & $15.3 \%$ \\
\hline Missing & 91 & $5.3 \%$ & 50 & $2.8 \%$ & 81 & $3.9 \%$ & 65 & $3.4 \%$ & 139 & $5.4 \%$ & 122 & $5.4 \%$ & 53 & $3.5 \%$ & 903 & $37.9 \%$ & 1504 & $9.3 \%$ \\
\hline Well l & & & & & & & & & & & & & & & & & & \\
\hline Low & 217 & $12.6 \%$ & 156 & $8.6 \%$ & 333 & $16.1 \%$ & 272 & $14.1 \%$ & 847 & $33.0 \%$ & 543 & $24.1 \%$ & 210 & $13.9 \%$ & 449 & $18.9 \%$ & 3027 & $18.7 \%$ \\
\hline Medium-low & 414 & $24.1 \%$ & 352 & $19.5 \%$ & 436 & $21.1 \%$ & 371 & $19.3 \%$ & 906 & $35.3 \%$ & 617 & $27.4 \%$ & 442 & $29.3 \%$ & 427 & $17.9 \%$ & 3965 & $24.4 \%$ \\
\hline Medium-high & 524 & $30.5 \%$ & 646 & $35.7 \%$ & 666 & $32.3 \%$ & 668 & $34.7 \%$ & 598 & $23.3 \%$ & 649 & $28.8 \%$ & 541 & $35.9 \%$ & 356 & $15.0 \%$ & 4648 & $28.6 \%$ \\
\hline High & 406 & $23.6 \%$ & 540 & $29.9 \%$ & 314 & $15.2 \%$ & 431 & $22.4 \%$ & 48 & $1.9 \%$ & 273 & $12.1 \%$ & 183 & $12.1 \%$ & 97 & $4.1 \%$ & 2292 & $14.1 \%$ \\
\hline Missing & 158 & $9.2 \%$ & 115 & $6.4 \%$ & 316 & $15.3 \%$ & 184 & $9.6 \%$ & 170 & $6.6 \%$ & 168 & $7.5 \%$ & 131 & $8.7 \%$ & 1051 & $44.2 \%$ & 2293 & $14.1 \%$ \\
\hline & & & & & & & & & & & & & & & & & & \\
\hline$\leq 7$ hours & 312 & $18.2 \%$ & 444 & $24.5 \%$ & 756 & $36.6 \%$ & 632 & $32.8 \%$ & 820 & $31.9 \%$ & 622 & $27.6 \%$ & 586 & $38.9 \%$ & 502 & $21.1 \%$ & 4674 & $28.8 \%$ \\
\hline 7 to $\leq 14$ hours & 603 & $35.1 \%$ & 839 & $46.4 \%$ & 699 & $33.8 \%$ & 732 & $38.0 \%$ & 937 & $36.5 \%$ & 817 & $36.3 \%$ & 554 & $36.8 \%$ & 805 & $33.8 \%$ & 5986 & $36.9 \%$ \\
\hline$>14$ hours & 717 & $41.7 \%$ & 446 & $24.7 \%$ & 448 & $21.7 \%$ & 466 & $24.2 \%$ & 706 & $27.5 \%$ & 721 & $32.0 \%$ & 288 & $19.1 \%$ & 625 & $26.3 \%$ & 4417 & $27.2 \%$ \\
\hline Missing & 87 & $5.1 \%$ & 80 & $4.4 \%$ & 162 & $7.8 \%$ & 96 & $5.0 \%$ & 106 & $4.1 \%$ & 90 & $4.0 \%$ & 79 & $5.2 \%$ & 448 & $18.8 \%$ & 1148 & $7.1 \%$ \\
\hline Junk rood & & & & & & & & & & & & & & & & & & \\
\hline Never & 121 & $7.0 \%$ & 276 & $15.3 \%$ & 30 & $1.5 \%$ & 26 & $1.3 \%$ & 227 & $8.8 \%$ & 184 & $8.2 \%$ & 126 & $8.4 \%$ & 84 & $3.5 \%$ & 1074 & $6.6 \%$ \\
\hline Occasionally & 778 & $45.3 \%$ & 1160 & $64.1 \%$ & 431 & $20.9 \%$ & 534 & $27.7 \%$ & 1132 & $44.1 \%$ & 831 & $36.9 \%$ & 810 & $53.7 \%$ & 673 & $28.3 \%$ & 6349 & $39.1 \%$ \\
\hline Often & 588 & $34.2 \%$ & 272 & $15.0 \%$ & 1369 & $66.3 \%$ & 1118 & $58.0 \%$ & 937 & $36.5 \%$ & 979 & $43.5 \%$ & 465 & $30.9 \%$ & 477 & $20.0 \%$ & 6205 & $38.2 \%$ \\
\hline Missing & 232 & $13.5 \%$ & 101 & $5.6 \%$ & 235 & $11.4 \%$ & 248 & $12.9 \%$ & 273 & $10.6 \%$ & 256 & $11.4 \%$ & 106 & $7.0 \%$ & 1146 & $48.2 \%$ & 2597 & $16.0 \%$ \\
\hline Soft drink availab & & & & & & & & & & & & & & & & & & \\
\hline Yes & 423 & $24.6 \%$ & 32 & $1.8 \%$ & 1060 & $51.3 \%$ & 486 & $25.2 \%$ & 579 & $22.5 \%$ & 321 & $14.3 \%$ & 64 & $4.2 \%$ & 158 & $6.6 \%$ & 3123 & $19.2 \%$ \\
\hline No & 1224 & $71.2 \%$ & 1690 & $93.4 \%$ & 896 & $43.4 \%$ & 1300 & $67.5 \%$ & 1892 & $73.6 \%$ & 1906 & $84.7 \%$ & 1395 & $92.6 \%$ & 930 & $39.1 \%$ & 11233 & $69.2 \%$ \\
\hline Missing & 72 & $4.2 \%$ & 87 & $4.8 \%$ & 109 & $5.3 \%$ & 140 & $7.3 \%$ & 98 & $3.8 \%$ & 23 & $1.0 \%$ & 48 & $3.2 \%$ & 1292 & $54.3 \%$ & 1869 & $11.5 \%$ \\
\hline
\end{tabular}


TABle 1: Continued.

\begin{tabular}{|c|c|c|c|c|c|c|c|c|c|c|c|c|c|c|c|c|c|c|}
\hline & \multicolumn{2}{|c|}{ Estonia } & \multicolumn{2}{|c|}{ Sweden } & \multicolumn{2}{|c|}{ Germany } & \multicolumn{2}{|c|}{ Belgium } & \multicolumn{2}{|c|}{ Hungary } & \multicolumn{2}{|c|}{ Italy } & \multicolumn{2}{|c|}{ Spain } & \multicolumn{2}{|c|}{ Cyprus } & \multicolumn{2}{|c|}{ All } \\
\hline & $n$ & $\%$ & $n$ & $\%$ & $n$ & $\%$ & $n$ & $\%$ & $n$ & $\%$ & $n$ & $\%$ & $n$ & $\%$ & $n$ & $\%$ & $n$ & $\%$ \\
\hline \multicolumn{19}{|l|}{ Sleep duration } \\
\hline$\leq 11$ hours & 1289 & $75.0 \%$ & 848 & $46.9 \%$ & 937 & $45.4 \%$ & 209 & $10.9 \%$ & 865 & $33.7 \%$ & 1578 & $70.1 \%$ & 464 & $30.8 \%$ & 908 & $38.2 \%$ & 7098 & $43.7 \%$ \\
\hline$>11$ hours & 42 & $2.4 \%$ & 366 & $20.2 \%$ & 649 & $31.4 \%$ & 199 & $10.3 \%$ & 36 & $1.4 \%$ & 64 & $2.8 \%$ & 40 & $2.7 \%$ & 45 & $1.9 \%$ & 1441 & $8.9 \%$ \\
\hline Missing & 388 & $22.6 \%$ & 595 & $32.9 \%$ & 479 & $23.2 \%$ & 1518 & $78.8 \%$ & 1668 & $64.9 \%$ & 608 & $27.0 \%$ & 1003 & $66.6 \%$ & 1427 & $60.0 \%$ & 7686 & $47.4 \%$ \\
\hline \multicolumn{19}{|l|}{ Item nonresponse } \\
\hline None & 1490 & $86.7 \%$ & 1644 & $90.9 \%$ & 1649 & $79.9 \%$ & 1628 & $84.5 \%$ & 2276 & $88.6 \%$ & 1907 & $84.8 \%$ & 1299 & $86.2 \%$ & 1150 & $48.3 \%$ & 13043 & $80.4 \%$ \\
\hline Low & 159 & $9.2 \%$ & 104 & $5.7 \%$ & 296 & $14.3 \%$ & 204 & $10.6 \%$ & 214 & $8.3 \%$ & 295 & $13.1 \%$ & 163 & $10.8 \%$ & 249 & $10.5 \%$ & 1684 & $10.4 \%$ \\
\hline High & 70 & $4.1 \%$ & 61 & $3.4 \%$ & 120 & $5.8 \%$ & 94 & $4.9 \%$ & 79 & $3.1 \%$ & 48 & $2.1 \%$ & 45 & $3.0 \%$ & 981 & $41.2 \%$ & 1498 & $9.2 \%$ \\
\hline Baseline response & \multicolumn{2}{|c|}{$54.5 \%$} & \multicolumn{2}{|c|}{$65.6 \%$} & \multicolumn{2}{|c|}{$48.2 \%$} & \multicolumn{2}{|c|}{$58.3 \%$} & \multicolumn{2}{|c|}{$43.7 \%$} & \multicolumn{2}{|c|}{$60.0 \%$} & \multicolumn{2}{|c|}{$41.4 \%$} & \multicolumn{2}{|c|}{$49.9 \%$} & \multicolumn{2}{|c|}{$51.4 \%$} \\
\hline
\end{tabular}

determinant variable includes a category which represents the missing values (non-response) for the respective item. Attrition proportions in the single categories of possible determinants ranged from $27.7 \%$ (children from highly educated parents) to $46.8 \%$ (children whose parents did not report their educational level). After control for country, age, and sex, the strongest impact on attrition was observed when baseline variable values were missing and children with nonresponse to certain items in T0 were about two times as likely to drop out of follow-up as children with information on these aspects. Thus, a high combined non-response score showed the highest impact of all potential determinants under investigation with a clear dose-effect relationship (low score: $\mathrm{OR}=1.45 ; 99 \%$ confidence interval (CI): 1.26, 1.68; high score: $\mathrm{OR}=2.63 ; 99 \% \mathrm{CI}: 2.10 ; 3.29)$.

Other, in particular sociodemographic and psychosocial factors were attributed to attrition as well. Hence, children from families where both parents were born in a foreign country were more likely to drop out as were children with parents of low educational background, while children from highly educated families showed a particularly high probability to participate in T1 (Table 2). Effects on attrition could be revealed also for some psychosocial aspects such as a low well-being score or a long screen time. Similarly, overweight and obese children participated less often in the followup examinations than normal weight children. In Model 2, age group, overweight/obesity, parental education level, migration background, family status, well-being, screen time, and the baseline proportionate participation were included. The non-response score variable was omitted from Model 2 to avoid overadjustment, since a missing category was already included for each of the variables in the model. The strongest effect on attrition was found for non-response regarding the items migration background, family status, and well-being. Generally, most of the effects (except for screen time) observed in model 1 persisted as statistically significant after multivariate adjustment although the associations were somewhat attenuated. Of note, sex, allocation in intervention or control region, dietary behaviour (junk food consumption and availability of soft drinks), sleep duration, and participation rate at baseline were not related to attrition in these models. Also considering baseline response as a continuous variable in sensitivity analysis, no correlation was found with proportionate attrition (Pearson's $r=-0.37 ; P=0.36$ ). Sensitivity analysis for model development applying forward selection of covariates resulted in the inclusion of the same variables as in the model based on the originally used selection procedure. The hierarchy for inclusion of the selected covariates was as follows: country, migration background, family status, well-being of the child, socioeconomic status, age group, and overweight.

Country-stratified comparisons regarding the main determinants for drop-out are given in Figure 2. No specific patterns or clustering of the determinants of attrition in the different countries were identified, indicating that the impact of determinants is not substantially stronger in centres with a high attrition proportion.

\section{Discussion}

Our results confirmed the hypothesis that overweight/obesity and factors related to obesity risk affect attrition to follow-up in a cohort study of children. This association was independent from differences in baseline proportionate participation and attrition to follow-up. The strongest effects in the adjusted models were seen for overweight/obesity and for item nonresponse at baseline, the latter especially with regard to migration background, family status, and well-being. A dose-effect relationship was found within the study centres between the numbers of item non-responses and the dropout. Generally, the extent of attrition does not seem to be related to the size of specific determinants for drop-out. The suggestion that selective drop-out due to a differential loss of individuals with specific patterns of obesity-related factors is stronger in study centres with high attrition proportions was thus not confirmed by our study. Sensitivity analysis excluding data from Spain and Belgium in Model 1 was conducted to account for the extremely high values in these countries with respect to the item non-response score. However, the results were only slightly attenuated but not essentially different (e.g., high non-response score: $\mathrm{OR}=2.15$; 99\% CI: 1.69, 2.73).

Determinants of attrition in longitudinal populationbased studies have been discussed in several publications $[3,19-21]$. In studies of young populations, where parental consent is usually required, sociodemographic factors, such as parental education or single parenthood, have been 
TABLE 2: Proportionate attrition and effect estimates for determinants of attrition in the IDEFICS study.

\begin{tabular}{|c|c|c|c|c|c|}
\hline & \multirow{2}{*}{ Proportionate attrition } & \multicolumn{2}{|c|}{ Model $1^{\mathrm{a}}$} & \multicolumn{2}{|c|}{ Model $2^{\mathrm{b}}$} \\
\hline & & OR & $99 \% \mathrm{CI}$ & OR & $99 \% \mathrm{CI}$ \\
\hline \multicolumn{6}{|l|}{ Sex } \\
\hline Male & $32.8 \%$ & Reference & & & \\
\hline Female & $32.3 \%$ & 0.97 & $0.89,1.06$ & & \\
\hline \multicolumn{6}{|l|}{ Age group } \\
\hline Preschool & $33.5 \%$ & 1.15 & $1.07,1.23$ & 1.22 & $1.11,1.34$ \\
\hline School & $31.8 \%$ & Reference & & Reference & \\
\hline \multicolumn{6}{|l|}{ Region } \\
\hline Intervention & $33.0 \%$ & Reference & & & \\
\hline Control & $32.1 \%$ & 0.96 & $0.88,1.05$ & & \\
\hline \multicolumn{6}{|l|}{ Weight status } \\
\hline Obese & $31.7 \%$ & 1.36 & $1.14,1.62$ & 1.28 & $1.07,1.54$ \\
\hline Overweight & $35.9 \%$ & 1.30 & $1.14,1.49$ & 1.27 & $1.10,1.45$ \\
\hline Normal weight & $36.8 \%$ & Reference & & Reference & \\
\hline \multicolumn{6}{|c|}{ Migration background } \\
\hline No & $31.0 \%$ & Reference & & Reference & \\
\hline Partly & $33.0 \%$ & 1.26 & $1.07,1.48$ & 1.23 & $1.04,1.45$ \\
\hline Full & $40.6 \%$ & 1.57 & $1.31,1.89$ & 1.15 & $0.91,1.45$ \\
\hline Missing & $46.6 \%$ & 2.44 & $1.98,3.00$ & 1.55 & $1.04,2.32$ \\
\hline \multicolumn{6}{|l|}{ Educational level } \\
\hline Low & $41.4 \%$ & 1.31 & $1.11,1.53$ & 1.19 & $1.01,1.40$ \\
\hline Medium-low & $34.0 \%$ & Reference & & Reference & \\
\hline Medium-high & $31.4 \%$ & 1.04 & $0.90,1.20$ & 1.06 & $0.92,1.22$ \\
\hline High & $27.7 \%$ & 0.84 & $0.75,0.94$ & 0.88 & $0.78,1.00$ \\
\hline Missing & $46.8 \%$ & 2.15 & $1.72,2.69$ & 1.09 & $0.72,1.68$ \\
\hline \multicolumn{6}{|l|}{ Family status } \\
\hline Two parents & $30.9 \%$ & Reference & & Reference & \\
\hline Single parent & $36.3 \%$ & 1.28 & $1.13,1.45$ & 1.21 & $1.06,1.38$ \\
\hline Missing & $40.0 \%$ & 1.96 & $1.66,2.32$ & 1.37 & $1.12,1.67$ \\
\hline \multicolumn{6}{|l|}{ Well-being } \\
\hline Low & $37.5 \%$ & 1.34 & $1.14,1.59$ & 1.26 & $1.07,1.49$ \\
\hline Medium-low & $33.0 \%$ & 1.18 & $1.00,1.38$ & 1.15 & $0.97,1.35$ \\
\hline Medium-high & $29.0 \%$ & 1.05 & $0.89,1.22$ & 1.04 & $0.89,1.22$ \\
\hline High & $25.4 \%$ & Reference & & Reference & \\
\hline Missing & $39.7 \%$ & 1.97 & $1.65,2.36$ & 1.46 & $1.19,1.79$ \\
\hline \multicolumn{6}{|c|}{ Weekly screen time } \\
\hline$\leq 7$ hours & $31.6 \%$ & Reference & & Reference & \\
\hline 7 to $\leq 14$ hours & $31.9 \%$ & 1.14 & $1.01,1.27$ & 1.11 & $0.99,1.24$ \\
\hline$>14$ hours & $31.9 \%$ & 1.16 & $1.03,1.32$ & 1.06 & $0.94,1.21$ \\
\hline Missing & $42.3 \%$ & 1.96 & $1.63,2.35$ & 1.10 & $0.86,1.39$ \\
\hline \multicolumn{6}{|l|}{ Junk food } \\
\hline Never & $29.0 \%$ & Reference & & & \\
\hline Occasionally & $29.7 \%$ & 1.00 & $0.83,1.23$ & & \\
\hline Often & $34.0 \%$ & 1.00 & $0.82,1.22$ & & \\
\hline Missing & $37.8 \%$ & 1.53 & $1.23,1.91$ & & \\
\hline \multicolumn{6}{|c|}{ Soft drink available } \\
\hline Yes & $38.7 \%$ & 1.11 & $0.98,1.25$ & & \\
\hline No & $30.0 \%$ & Reference & & & \\
\hline Missing & $37.3 \%$ & 1.97 & $1.66,2.33$ & & \\
\hline
\end{tabular}


TABLE 2: Continued.

\begin{tabular}{|c|c|c|c|c|c|}
\hline & \multirow{2}{*}{ Proportionate attrition } & \multicolumn{2}{|c|}{ Model $1^{a}$} & \multicolumn{2}{|c|}{ Model $2^{\mathrm{b}}$} \\
\hline & & OR & $99 \% \mathrm{CI}$ & OR & $99 \% \mathrm{CI}$ \\
\hline \multicolumn{6}{|l|}{ Sleep duration } \\
\hline$\leq 11$ hours & $29.0 \%$ & 0.86 & $0.72,1.03$ & & \\
\hline$>11$ hours & $25.5 \%$ & Reference & & & \\
\hline Missing & $35.3 \%$ & 1.08 & $0.91,1.29$ & & \\
\hline \multicolumn{6}{|c|}{ Item non-response } \\
\hline No missing & $30.8 \%$ & Reference & & & \\
\hline Low & $39.0 \%$ & 1.45 & $1.26,1.68$ & & \\
\hline High & $40.4 \%$ & 2.18 & $1.83,2.58$ & & \\
\hline \multicolumn{6}{|c|}{ Baseline participation } \\
\hline Low & $27.3 \%$ & 1.50 & $0.54,4.16$ & 1.33 & $0.46,3.89$ \\
\hline High & $37.3 \%$ & Reference & & Reference & \\
\hline
\end{tabular}

CI: confidence interval; OR: odds ratio.

${ }^{a}$ Adjusted for country, sex, and age group.

${ }^{\mathrm{b}}$ Adjusted for country, age group, weight status, parental education level, migration background, family status, well-being, screen time, and baseline proportionate participation.

reported as the most relevant attrition determinants [4, 22]. These reports are in line with our results, where socio-demographic aspects including parental education, single parenthood, and migration background also affected nonparticipation in the follow-up study. This suggests that during the conduct of follow-up studies, special attention has to be paid to these groups in order to optimize their study adherence.

Behavioural factors, such as sedentary behaviour or diet, were not or only weakly associated with attrition, which corroborates findings from the Danish Youth Cohort study [3]. However, our data revealed an impact of the children's wellbeing on attrition. To the authors' knowledge no such association has been previously reported in other studies of young populations. The same applies to the impact of item nonresponse, in particular to the dose-effect relationship between the number of item non-responses and attrition that was observed in the analysis. This seems to suggest that parents who were reluctant to completely answer the questionnaire or to participate in the respective study modules already at baseline were more likely to not participate after two years. In this context, results from a German cohort should be noted where late invitation response and the intensity of recruitment efforts were determinants of attrition to follow-up [2]. The authors suppose that late response is just an indicator for reluctance to participation and thus probably associated with a high value of the item non-response scores. Unfortunately, local variations in the organization of recruitment procedures in the different study centres and the different settings in each country precluded more detailed analyses of recruitment efforts. Nevertheless, intensified motivation procedures may need to be considered for participants in the cohort panel who show high item non-response scores.

In previous studies it has been discussed that individuals might select themselves out of studies due to treatment or intervention [23]. Interestingly, no differences in attrition were seen with regard to being allocated to the intervention or the control region in the present study, which may indicate that the IDEFICS intervention programme was either well accepted or so low threshold that the decision to take part in the follow-up was unaffected.

An important aspect in this context is the potential for biased estimates in longitudinal analyses due to selective attrition. Selective attrition threatens to impair the generalizability of findings and to bias the estimates of associations [24]. Although evidence for this is limited and conflicting [2, 19], this aspect needs to be considered in the interpretation of our results, in particular regarding how the systematic drop-out in the present study threatens to induce bias in the longitudinal analysis of certain outcomes. Particularly the elevated probability of drop-out for overweight or obese children, or with factors related to the development of overweight, is of particular concern here. Thus, in a prospective analysis using the incidence of obesity as a dichotomous outcome, the differential drop-out of children in the overweight (and even more so in the upper overweight) category, where the progression to obesity is more likely to occur than in children with a lower BMI, introduces an elevated probability of missing the outcome of interest in the follow-up examinations; this might lead to a biased estimation of the association under investigation. Therefore, alternative analytic approaches, for example, by using the relative change in BMI, and thereby identifying changes in the BMI within the whole range of the BMI distribution, as the outcome of interest, could be one approach to diminish the observed limitations in this cohort.

Another example, where the selective drop-out might impair longitudinal analysis, is the study of the incidence of secondary diseases that are more likely to occur in overweight or obese children, for example, type 2 diabetes [25]. The elevated drop-out of children from this group could lead to a relevant loss of statistical power as the number of incident cases may become too low to observe significant effects since these conditions are generally rare (although increasing) within the age group of interest [26]. We suppose that the prospective analysis of observational studies within the context 


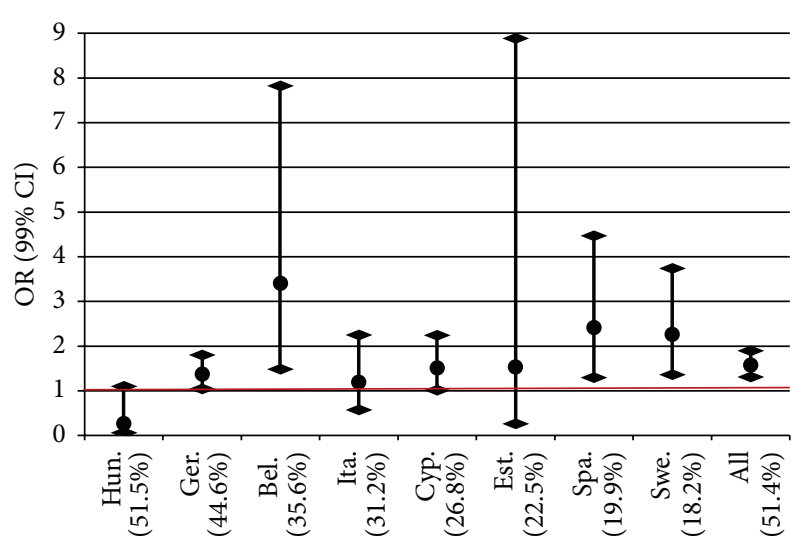

(a) Migration background

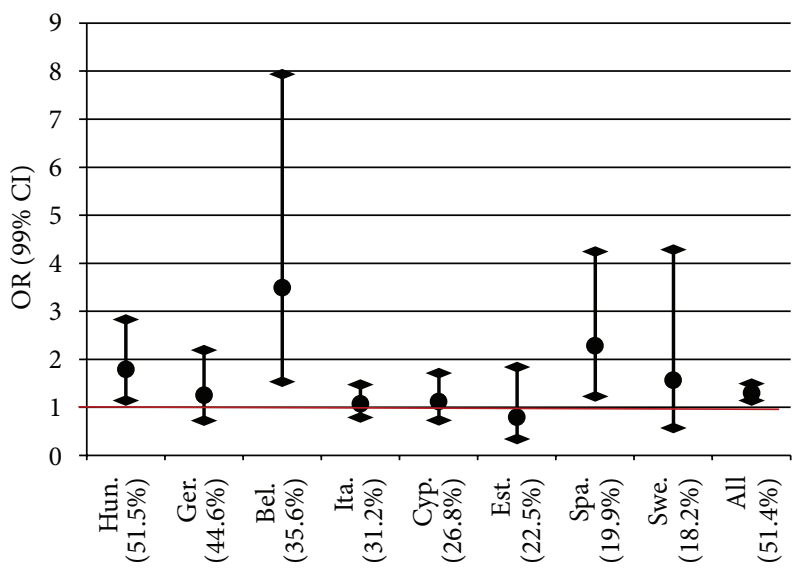

(c) Overweight

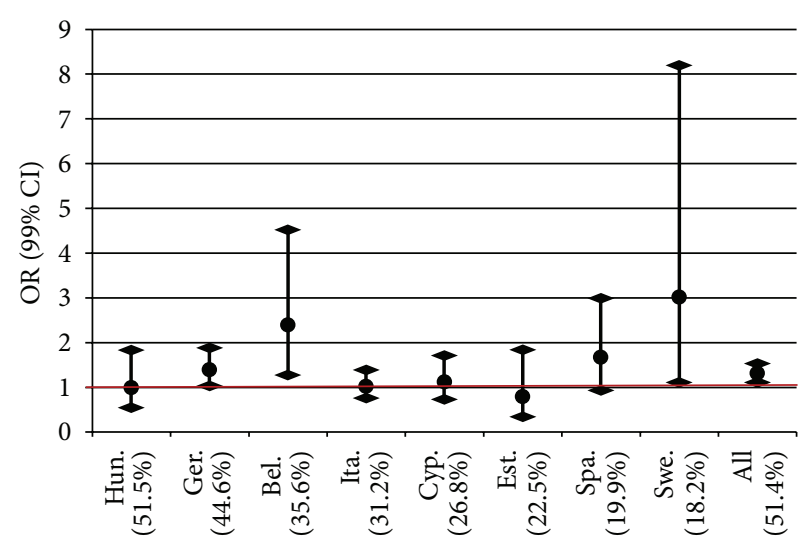

(b) Low education

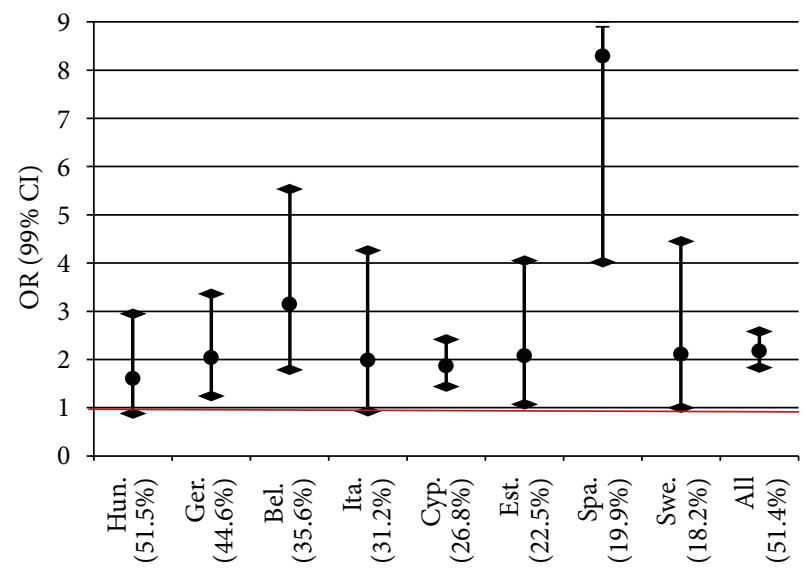

(d) Item-Non-Response

FIGURE 2: Effect estimates (OR, 99\% CI) for the main determinants by country (ordered by the extent of proportionate attrition).

of obesity research in children should conscientiously take these aspects into consideration. While the results concerning obesity-related determinants of attrition cannot be generalized to cohort studies with a different research focus, the identification of item non-response as the factor with the strongest impact might be relevant for most longitudinal studies conducted. This special population group can be identified based on baseline data and extensive recruitment strategies might be developed and applied in order to increase study retention among this population.

Data of the reasons for attrition (lack of interest, illness, lack of time, etc.) would have deepened the analyses in this report. However, no or only partial information on reasons for loss to follow-up was assessed during the field work and therefore no such analysis was feasible in the context of the present study.

\section{Conclusion}

Our results indicate selective attrition in cohort studies of children which are independent from baseline participation in the study centres. The main determinants of loss to follow-up were a high level of item non-response at baseline, especially lack of information on sociodemographic and psychosocial factors, followed by children's overweight and disadvantageous sociodemographic and psychosocial conditions. Exposure or nonexposure to the intervention programme did not affect participation in follow-up examinations. Observational studies of obesity research in children should provide for these aspects in the longitudinal analysis of data, but preferably already during the planning and conduct of follow-up surveys.

\section{Conflict of Interests}

The authors declare that they have no conflict of interests.

\section{Acknowledgments}

This work was funded by the European Community within the Sixth RTD Framework Programme Contract no. 016181 (FOOD) and was done as part of the IDEFICS Study (http://www.idefics.eu/). The authors are grateful for the support by school boards, headmasters, and communities. The information in this paper reflects the author's view and is provided as is (on behalf of the European Consortium of the IDEFICS Project (http://www.idefics.eu/)). 


\section{References}

[1] F. Lamers, A. W. Hoogendoorn, J. H. Smit, D. R. van, F. G. Zitman, W. A. Nolen et al., "Sociodemographic and psychiatric determinants of attrition in the Netherlands study of depression and anxiety (NESDA)," Comprehensive psychiatry, vol. 53, no. 1, pp. 63-70, 2012.

[2] R. Haring, D. Alte, H. Völzke et al., "Extended recruitment efforts minimize attrition but not necessarily bias," Journal of Clinical Epidemiology, vol. 62, no. 3, pp. 252-260, 2009.

[3] M. Vinther-Larsen, M. Riegels, M. H. Rod, M. Schiøtz, T. Curtis, and M. Grønbaek, "The danish youth cohort: characteristics of participants and non-participants and determinants of attrition," Scandinavian Journal of Public Health, vol. 38, no. 6, pp. 648-656, 2010.

[4] P. M. Zook, C. Jordan, B. Adams et al., "Retention strategies and predictors of attrition in an urban pediatric asthma study," Clinical Trials, vol. 7, no. 4, pp. 400-410, 2010.

[5] J. P. Rey-López, G. Vicente-Rodríguez, M. Biosca, and L. A. Moreno, "Sedentary behaviour and obesity development in children and adolescents," Nutrition, Metabolism and Cardiovascular Diseases, vol. 18, no. 3, pp. 242-251, 2008.

[6] S. Hense, H. Pohlabeln, S. De Henauw et al., "Sleep duration and overweight in European children: is the association modified by geographic region?" Sleep, vol. 34, no. 7, pp. 885-890, 2011.

[7] L. Johnson, A. P. Mander, L. R. Jones, P. M. Emmett, and S. A. Jebb, "A prospective analysis of dietary energy density at age 5 and 7 years and fatness at 9 years among UK children," International Journal of Obesity, vol. 32, no. 4, pp. 586-593, 2008.

[8] T. A. McCaffrey, K. L. Rennie, M. A. Kerr et al., "Energy density of the diet and change in body fatness from childhood to adolescence; is there a relation?" American Journal of Clinical Nutrition, vol. 87, no. 5, pp. 1230-1237, 2008.

[9] J. Williams, M. Wake, K. Hesketh, E. Maher, and E. Waters, "Health-related quality of life of overweight and obese children," Journal of the American Medical Association, vol. 293, no. 1, pp. 70-76, 2005.

[10] W. Ahrens, K. Bammann, A. Siani, K. Buchecker, H. S. de, L. Iacoviello et al., "The IDEFICS cohort: design, characteristics and participation in the baseline survey," International Journal of Obesity, vol. 35, supplement 1, pp. S3-S15, 2011.

[11] S. de Henauw, V. Verbstel, S. Marild, G. Barba, K. Bammann, G. Eiben et al., "The IDEFICS community-oriented intervention programme: a new model for childhood obesity prevention in Europe?" International Journal of Obesity, vol. 35, supplement 1, pp. S16-S23, 2011.

[12] K. Bammann and W. Ahrens, Measurement Tools for a Health Survey on Nutrition, Physical Activity and Lifestyle in Children: the European IDEFICS Study, Springer, New York, NY, USA, 2013.

[13] T. J. Cole, M. C. Bellizzi, K. M. Flegal, and W. H. Dietz, "Establishing a standard definition for child overweight and obesity worldwide: international survey," British Medical Journal, vol. 320, no. 7244, pp. 1240-1243, 2000.

[14] M. Bullinger, A. L. Brütt, M. Erhart, and U. Ravens-Sieberer, "Psychometric properties of the KINDL-R questionnaire: results of the BELLA study," European Child and Adolescent Psychiatry, vol. 17, no. 1, pp. 125-132, 2008.

[15] U. Ravens-Sieberer and M. Bullinger, Measuring Health Related Quality of Life in Children and Adolescents, 2000.

[16] S. Bel-Serrat, T. Mouratidou, V. Pala, I. Huybrechts, C. Bornhorst, J. M. Fernandez-Alvira et al., "Relative validity of the children's eating habits questionnaire-food frequency section among young European children: the IDEFICS study," Public Health Nutrition, pp. 1-11, 2013.

[17] A. Lanfer, A. Hebestreit, W. Ahrens et al., "Reproducibility of food consumption frequencies derived from the children's eating habits questionnaire used in the IDEFICS study," International Journal of Obesity, vol. 35, supplement 1, pp. S61-S68, 2011.

[18] S. Hense, G. Barba, H. Pohlabeln et al., "Factors that influence weekday sleep duration in European children," Sleep, vol. 34, no. 5, pp. 633-639, 2011.

[19] C. L. Booker, S. Harding, and M. Benzeval, "A systematic review of the effect of retention methods in population-based cohort studies," BMC Public Health, vol. 11, article 249, 2011.

[20] S. A. Fröjd, R. Kaltiala-Heino, and M. J. Marttunen, "Does problem behaviour affect attrition from a cohort study on adolescent mental health?" European Journal of Public Health, vol. 21, no. 3, pp. 306-310, 2011.

[21] I. Moroshko, L. Brennan, and P. O’Brien, "Predictors of dropout in weight loss interventions: a systematic review of the literature," Obesity Reviews, vol. 12, pp. 912-934, 2011.

[22] D. Wolke, A. Waylen, M. Samara et al., "Selective drop-out in longitudinal studies and non-biased prediction of behaviour disorders," British Journal of Psychiatry, vol. 195, no. 3, pp. 249256, 2009.

[23] J. S. Goodman and T. C. Blum, "Assessing the non-random sampling effects of subject attrition in longitudinal research," Journal of Management, vol. 22, no. 4, pp. 627-652, 1996.

[24] L. Marcellus, "Are we missing anything? Pursuing research on attrition," Canadian Journal of Nursing Research, vol. 36, no. 3, pp. 82-98, 2004.

[25] S. Dhuper, S. Buddhe, and S. Patel, "Managing cardiovascular risk in overweight children and adolescents," Paediatr Drugs. In press.

[26] A. D. Liese, R. B. D’Agostino Jr, R. F. Hamman et al., "The burden of diabetes mellitus among US youth: prevalence estimates from the SEARCH for diabetes in youth study," Pediatrics, vol. 118, no. 4, pp. 1510-1518, 2006. 


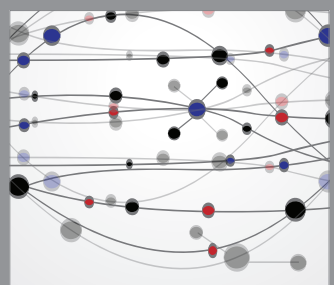

The Scientific World Journal
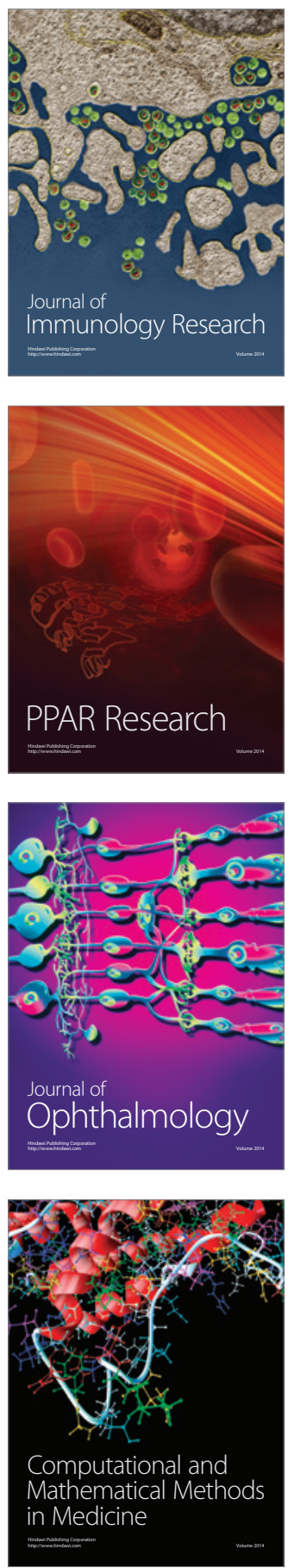

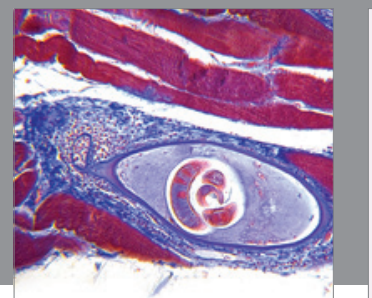

Gastroenterology

Research and Practice
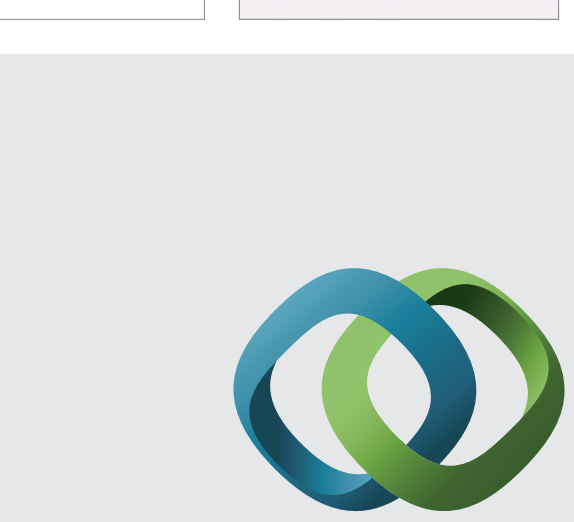

\section{Hindawi}

Submit your manuscripts at

http://www.hindawi.com
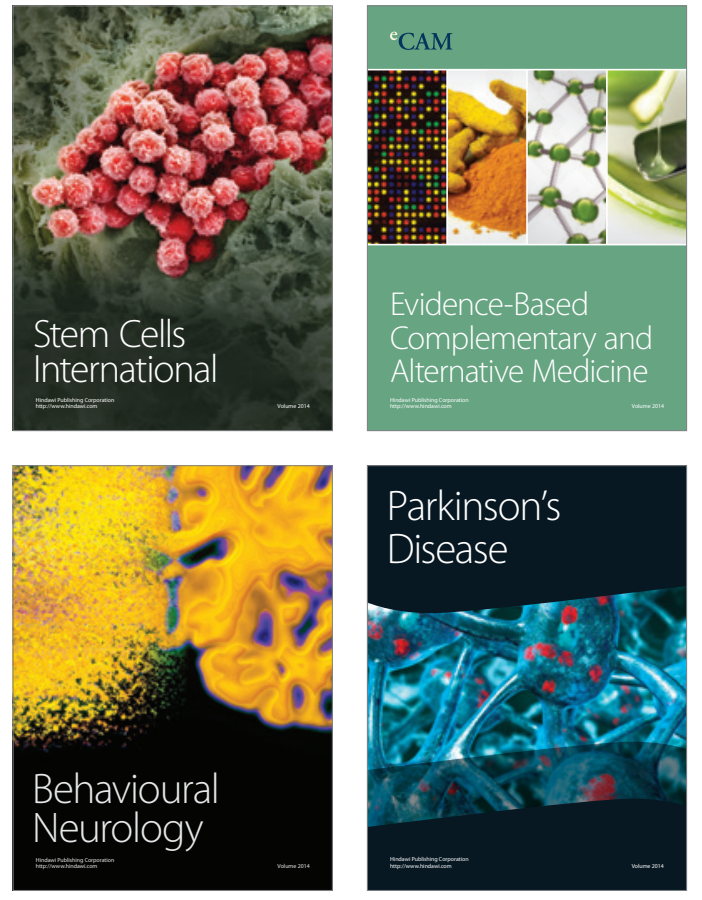
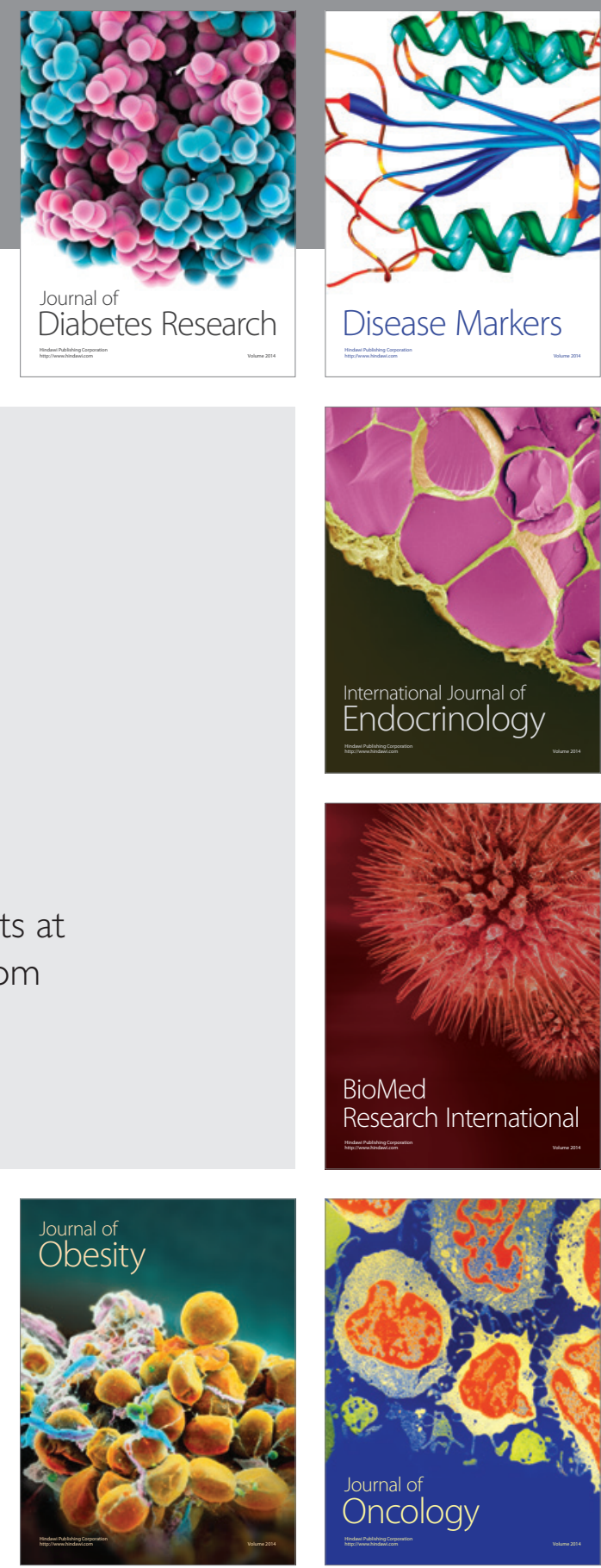

Disease Markers
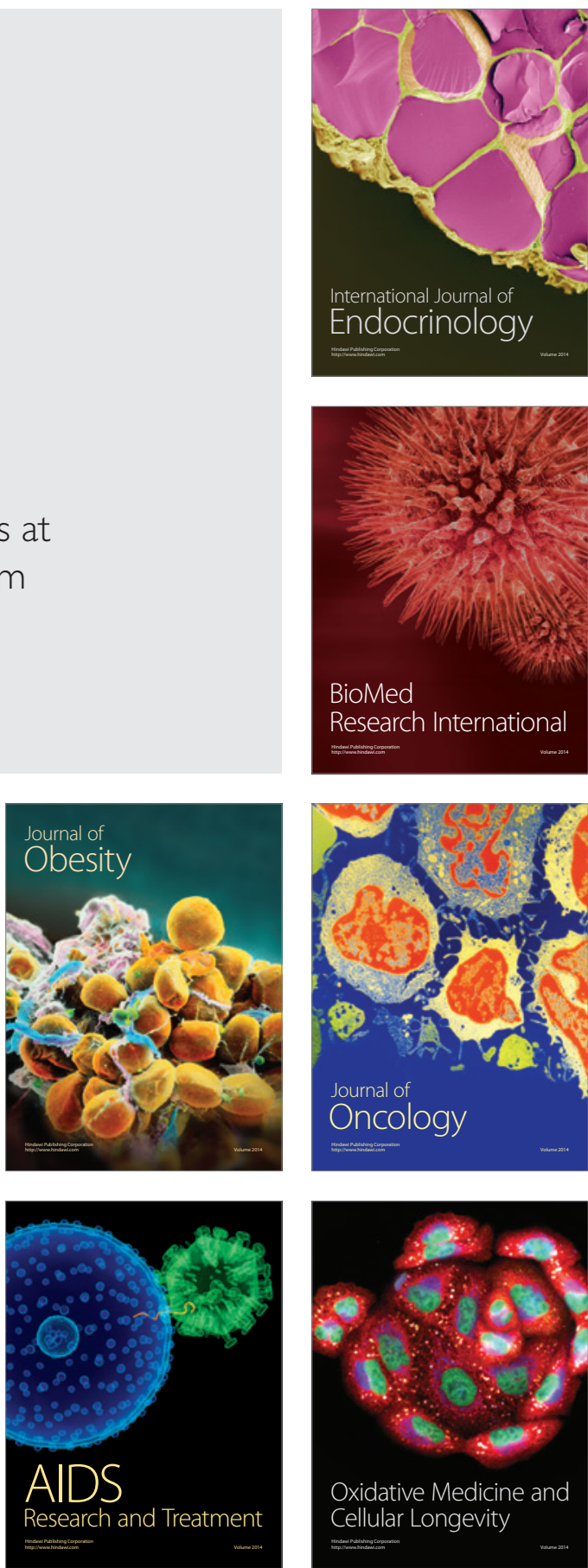